\title{
Spatial and seasonal variations of leaf area index (LAI) in subtropical secondary forests related to floristic composition and stand characters
}

Wenjuan Zhu et al.

Correspondence to: Wenhua Xiang (xiangwh2005@163.com)

The copyright of individual parts of the supplement might differ from the CC-BY 3.0 licence. 
Table S1 Stand density, average diameter at breast height (DBH), average height $(\mathrm{H})$, basal area (BA) and important value (IV) of the top five tree species, others and whole stand in P. massoniana-L. glaber, C. axillaris and L. glaber-Cyclobalanopsis glauca forests. Values in parenthesis are the range of DBH 5 and $\mathrm{H}$.

\begin{tabular}{lllllll}
\hline Forest type & Tree species & $\begin{array}{l}\text { Density } \\
\left(\mathrm{stem} \cdot \mathrm{h}^{-1}\right)\end{array}$ & $\mathrm{DBH}(\mathrm{cm})$ & $\mathrm{H}(\mathrm{m})$ & $\begin{array}{l}\mathrm{BA} \\
\left(\mathrm{m}^{2} \cdot \mathrm{ha}^{-1}\right)\end{array}$ & $\mathrm{IV}$ \\
\hline & P. massoniana & 495 & $14.4(4.0-42.7)$ & $12.5(1.6-25.1)$ & 9.45 & 28.24 \\
& L. glaber & 566 & $7.2(4.0-25.0)$ & $7.4(1.7-18.4)$ & 2.76 & 20.04 \\
P. massoniana-L. & Cinnamomum camphora & 159 & $15.1(4.4-37.7)$ & $10.8(2.0-18.2)$ & 3.40 & 10.64 \\
glaber & C. glauca & 126 & $10.3(4.0-27.5)$ & $7.8(1.8-19.4)$ & 1.43 & 7.51 \\
& Liquidambar formosana & 84 & $13.4(4.2-33.7)$ & $10.1(3.6-18.3)$ & 1.56 & 6.34 \\
& Others & 407 & $8.5(4.0-37.2)$ & $7.3(2.1-22.5)$ & 3.47 & 27.23 \\
& Whole stand & 1837 & $10.6(4.0-42.7)$ & $9.2(1.6-25.1)$ & 22.07 & 100.00 \\
\hline \multirow{5}{*}{ C. axillaris } & C. axillaris & 184 & $23.3(4.1-54.1)$ & $15.7(3.2-34.5)$ & 9.12 & 26.49 \\
& Loropetalum chinensis & 401 & $5.2(4.0-14.8)$ & $4.9(1.6-21.5)$ & 0.89 & 15.51 \\
& Symplocos setchuensis & 144 & $6.4(4.0-20.3)$ & $5.2(2.0-8.9)$ & 0.55 & 6.72 \\
& Vernicia Montana & 57 & $10.4(4.0-41.8)$ & $9.7(1.5-23.6)$ & 0.85 & 4.99 \\
& Vernicia fordii & 44 & $11.4(4.2-33.3)$ & $10.0(3.6-23.5)$ & 0.59 & 3.91 \\
& Others & 600 & $7.9(4.0-41.8)$ & $6.2(1.4-32.5)$ & 4.63 & 42.38 \\
& Whole stand & 1430 & $9.2(4.0-54.1)$ & $7.2(1.3-34.5)$ & 16.63 & 100.00 \\
\hline L.glaber-C. & L. glaber & 586 & $10.4(4.0-37.1)$ & $9.6(2.2-19.5)$ & 6.70 & 25.93 \\
glauca & C. glauca & 164 & $12.8(4.0-34.6)$ & $10.4(3.8-20.0)$ & 3.01 & 9.90 \\
& P. massoniana & 123 & $18.0(7.0-32.2)$ & $14.2(1.3-20.0)$ & 3.46 & 9.77 \\
& C. axillaris & 83 & $19.3(4.0-46.8)$ & $13.5(1.8-20.2)$ & 2.85 & 7.91 \\
& Cleyera japonica & 199 & $5.9(4.0-18.2)$ & $5.8(2.2-16.1)$ & 0.61 & 7.42 \\
& Others & 633 & $10.2(4.0-40.0)$ & $9.5(1.5-21.0)$ & 7.04 & 39.07 \\
& Whole stand & 1788 & $11.0(4.0-46.8)$ & $9.7(1.3-21.0)$ & 23.67 & 100.00 \\
\hline
\end{tabular}

